\title{
Metastable Defect Formation at Microvoids Identified as a Source of Light-Induced Degradation in $a-S i: H$
}

\author{
M. Fehr, ${ }^{1, *}$ A. Schnegg, ${ }^{1, \dagger}$ B. Rech, ${ }^{1}$ O. Astakhov, ${ }^{2}$ F. Finger, ${ }^{2}$ R. Bittl,${ }^{3}$ C. Teutloff, ${ }^{3}$ and K. Lips ${ }^{1}$ \\ ${ }^{1}$ Helmholtz-Zentrum Berlin für Materialien und Energie, Institut für Silizium-Photovoltaik, Kekuléstr. 5, 12489 Berlin, Germany \\ ${ }^{2}$ Forschungszentrum Jülich, Institut für Energie- und Klimaforschung, Photovoltaik, 52425 Jülich, Germany \\ ${ }^{3}$ Freie Universität Berlin, Fachbereich Physik, Arnimallee 14, 14195 Berlin, Germany
}

(Received 28 August 2013; published 12 February 2014)

\begin{abstract}
Light-induced degradation of hydrogenated amorphous silicon $(a-\mathrm{Si}: \mathrm{H})$, known as the StaeblerWronski effect, has been studied by time-domain pulsed electron-paramagnetic resonance. Electron-spin echo relaxation measurements in the annealed and light-soaked state revealed two types of defects (termed type I and II), which can be discerned by their electron-spin echo relaxation. Type I exhibits a monoexponential decay related to indirect flip-flop processes between dipolar coupled electron spins in defect clusters, while the phase relaxation of type II is dominated by ${ }^{1} \mathrm{H}$ nuclear spin dynamics and is indicative for isolated spins. We propose that defects are either located at internal surfaces of microvoids (type I) or are isolated and uniformly distributed in the bulk (type II). The concentration of both defect type I and II is significantly higher in the light-soaked state compared to the annealed state. Our results indicate that in addition to isolated defects, defects on internal surfaces of microvoids play a role in light-induced degradation of device-quality $a-\mathrm{Si}: \mathrm{H}$.
\end{abstract}

DOI: 10.1103/PhysRevLett.112.066403

PACS numbers: 71.55.Jv, 76.30.-v, 88.40.jj

The efficiency of photovoltaic energy conversion from sunlight to electricity in devices based on hydrogenated amorphous silicon $(a-\mathrm{Si}: \mathrm{H})$ is reduced by light-induced degradation. Exposure to intense sunlight for prolonged time decreases the efficiency of $a-\mathrm{Si}: \mathrm{H}$ solar cells by up to $30 \%$, an effect that is referred to as the Staebler-Wronski effect (SWE) $[1,2]$. Recombination of excess electron-hole pairs generated by illumination creates additional coordination defects, which induce electronic states in the middle of the band gap. The enhanced recombination via those defect states causes a degradation of solar cell performance [3]. Light-induced defects are metastable in the sense that they can be removed by annealing at moderate temperatures $\left(100-200^{\circ} \mathrm{C}\right.$ for $\left.1-2 \mathrm{~h}\right)$ [3,4], while defects present after deposition (here referred to as native) are stable with respect to annealing up to the deposition temperature. In undoped $a-\mathrm{Si}: \mathrm{H}$, electron-paramagnetic resonance (EPR) indicates that native as well as metastable defects are threefold-coordinated $\mathrm{Si}$ atoms [dangling bonds (DB)] with a remaining unpaired electron, and, thus, are paramagnetic [3-6]. Although a wide variety of models was proposed to explain the SWE [7-12], its microscopic process has remained unclear [11,12]. Most models of the SWE are built on recombination-induced breaking of weak Si-Si bonds $[3,11]$ or formation of metastable $\mathrm{H}$ complexes [13] assuming a uniform defect distribution in a continuous random network of $\mathrm{Si}$ and $\mathrm{H}$ atoms. However, it has been shown that $a-\mathrm{Si}: \mathrm{H}$, regardless of its structural and electronic quality, is inhomogeneous and contains a large amount of microvoids and vacancies [14-18]. In $a-\mathrm{Si}: \mathrm{H}$, nuclear-magnetic resonance [19,20], H effusion [18,21], positron-annihilation spectroscopy [22], and Fourier-transform infrared spectroscopy [23] indicate the presence of $\mathrm{H}$ clusters and $\mathrm{H}$ passivated multivacancies (2 to 7 missing Si atoms). In addition, small-angle $\mathrm{x}$-ray scattering experiments suggest that, even in the case of device-quality films, $a$-Si: $\mathrm{H}$ contains microvoids of $\approx 1 \mathrm{~nm}$ diameter at a concentration of $\approx 10^{19} \mathrm{~cm}^{-3}$ [15]. Further studies presented empirical evidence that the amount of microvoids is correlated to solar cell performance in the initial and light-degraded state [24,25], indicating that microvoids play a role in light-induced degradation. A corresponding model explaining the SWE relying on $\mathrm{H}$ migration on internal surfaces of microvoids has been presented by Carlson [26]. However, experimental evidence for a microscopic link between microvoids and metastable defect creation is missing so far.

In this work, we present results from time-domain pulsed EPR spectroscopy providing evidence that a significant portion of metastable defects form dense clusters. We propose that these clusters are linked to microvoids and their inner surfaces. Since metastable defects in undoped $a-\mathrm{Si}: \mathrm{H}$ are paramagnetic and carry an electron spin, details on the atomic length scale can be inferred by EPR. This technique resolves interactions between electron spins (i.e., defect sites) and also between electron and nuclear spins (e.g., $\left.{ }^{1} \mathrm{H}\right)$. Those interactions are important, since the spatial distribution of defects and nuclei is encoded in their dipolar and isotropic part. While spin-spin interactions are usually too small to be resolved in the EPR spectrum, they often dominate the relaxation of electron-spin echoes (ESEs) in solids [27-31]. In pulsed EPR, the excitation bandwidth is usually narrow compared to the width of the EPR spectrum and we can divide spins into excited $(A)$ 
and nonexcited spins $(B) . B$ spins are subject to random fluctuations induced either by spin-lattice relaxation or mutual spin flip flops (spin diffusion). These fluctuations randomly shift the resonance frequency of $A$ spins causing a loss in phase coherence, a process referred to as spectral diffusion (SD).

Here, we measure the ESE relaxation of defect states, which allows us to infer details about their spatial distribution. We found that $a-\mathrm{Si}: \mathrm{H}$ in the light-soaked and annealed state contains two types of defects, which differ in their ESE relaxation. We discuss their microscopic environment and propose that defects of type I are clustered at microvoids giving rise to a fast ESE decay while defects of type II are randomly distributed, resulting in a less rapid ESE decay dominated by ${ }^{1} \mathrm{H}$ nuclear spin dynamics.

Undoped device-grade $a-\mathrm{Si}: \mathrm{H}$ and $a-\mathrm{Si}: \mathrm{D}$ films of $1 \mu \mathrm{m}$ thickness were deposited with plasma-enhanced chemical vapor deposition (PECVD) on $\mathrm{Al}$ foil $[32,33]$. The samples where light soaked for $760-1000 \mathrm{~h}$ at $50^{\circ} \mathrm{C}$. We applied filtered (cutoff $550 \mathrm{~nm}$ ) AM1.5 light to avoid highly nonuniform defect distributions, which were observed in earlier studies applying white light [34]. The defect density in light-soaked $a-\mathrm{Si}: \mathrm{H}$ is $3.2 \times 10^{16} \mathrm{~cm}^{-3}$ and is reduced to $5.8 \times 10^{15} \mathrm{~cm}^{-3}$ after annealing samples in sealed quartz tubes at $180^{\circ} \mathrm{C}$ for $14 \mathrm{~h}$. Similar values are obtained for $a$-Si:D. Field-swept echoes (FSEs) and ESE decays were measured with a two-pulse ESE sequence $(\pi / 2-\tau-\pi-$ $\tau$-echo) at $T=60 \mathrm{~K}$. Experiments were carried out at a microwave frequency of $34 \mathrm{GHz}$ providing higher sensitivity compared to earlier studies operating at $9 \mathrm{GHz}$ [35]. Details about samples and methods are presented in the Supplemental Material [36]. FSE-EPR spectra and ESE decays of light soaked $a-\mathrm{Si}: \mathrm{H}$ are shown in Figs. 1(a) and 1(b), respectively. Most strikingly we observe that the overall ESE decays cannot be described by a stretchedexponential decay $\exp ^{-\left(\tau / T_{\mathrm{SD}}\right)^{n}}$ over the full $\tau$ range and, in addition, depend on magnetic-field position $\left(B_{0}\right)$, indicated by arrows in Fig. 1(a). We will show that ESE decays in the light-soaked [Fig. 1(b)] as well as in the annealed state [Fig. 1(c)] consist of a superposition of two types of defects that differ in their phase relaxation and EPR spectrum. The ESE decay of defect type I is fast and monoexponential ( $n=1$, best seen in the initial $\tau$ regime: $2 \tau<5 \mu$ s) with a time constant $T_{\mathrm{SD}, \mathrm{I}}=2.4(1) \mu \mathrm{s}$. The ESE decay of defect type II is slow and follows an $\exp ^{-\left(\tau / T_{\mathrm{SD}, \mathrm{II}}\right)^{2}}$ law (best seen in the long $\tau$ regime: $2 \tau>5 \mu \mathrm{s}$ ) with $T_{\mathrm{SD}, \mathrm{II}}=11.4(1) \mu \mathrm{s}$. $T_{\mathrm{SD}, \mathrm{I}(\mathrm{II})}$ are temperature independent, as described in the Supplemental Material [36]. Our model is summarized in the following formula describing the ESE signal $V$ as a function of $\tau$ and $B_{0}$ :

$$
V\left(\tau, B_{0}\right)=A_{\mathrm{I}}\left(B_{0}\right) e^{-2 \tau / T_{\mathrm{SD}, \mathrm{I}}}+A_{\mathrm{II}}\left(B_{0}\right) e^{-\left(\tau / T_{\mathrm{SD}, \mathrm{II}}\right)^{2}} .
$$

Here, $A_{\mathrm{I}, \mathrm{II}}\left(B_{0}\right)$ describe amplitudes of individual EPR spectra. Equation (1) provides excellent fits to the experimental data [see the red lines in Figs. 1(b) and 1(c)]. Our assumption about a superposition of two defect types is supported by the effect of isotope exchange $\left({ }^{1} \mathrm{H} \rightarrow{ }^{2} \mathrm{D}\right)$ on ESE decays. While FSE spectra are unchanged [see Figs. 1(a) and 1(d)], Figs. 1(e) and 1(f) show that replacing ${ }^{1} \mathrm{H}$ with ${ }^{2} \mathrm{D}$ drastically influences the ESE decay. While the relaxation rate of defect type II (slow decay) is dramatically reduced, the rate of type I (fast decay) varies only slightly. A spectral decomposition of the ESE decays for $a$-Si:D relying on a formula similar to Eq. (1) finds $T_{\mathrm{SD}, \mathrm{I}}=$ 3.8(1) $\mu$ s (see the Supplemental Material [36]). This finding supports the presence of a superposition of two independent defect types. Furthermore, isotope exchange shows that the origin of spin relaxation for defect type II is ${ }^{1} \mathrm{H}$ nuclear spin induced SD (see also the Supplemental Material [36]). ${ }^{2} \mathrm{D}$ carries a smaller magnetic moment compared to ${ }^{1} \mathrm{H}$ which leads to a smaller magnetic field felt by the electron spin and, therefore, increases $T_{\mathrm{SD}, I I}$ [37]. A more careful analysis of the ESE decay of defect type II in $a-\mathrm{Si}$ : D shows that instead of $\mathrm{SD}$, instantaneous diffusion (ID) is the limiting process (see the Supplemental Material [36]). From the ID rate constant we can infer that type II defects in $a$-Si:D are uniformly distributed [38]; i.e., defects are isolated from each other. This finding should also hold for $a-\mathrm{Si}: \mathrm{H}$.

As shown in Fig. 1(b), ESE decays of $a$-Si: $\mathrm{H}$ depend on $B_{0}$, which is equivalent to a dependence of FSE-EPR spectra on $\tau$. This dependence has been measured for consistency [see Fig. 2(a)] showing that FSE-EPR spectra increase in width as $\tau$ is increased. This implies that EPR spectra of defect type I and II $\left(A_{\mathrm{I}, \mathrm{II}}\right)$ differ, which is shown in Fig. 2(b) for the light-soaked state of $a-\mathrm{Si}: \mathrm{H}$. Equivalent results are obtained for the annealed state of $a-\mathrm{Si}: \mathrm{H}$. A spectral decomposition for the $a-\mathrm{Si}: \mathrm{D}$ sample is discussed in the Supplemental Material [36].

The defect density in the light-soaked state is higher than in the annealed state, which is due to a change in concentration of both defect types. In order to quantify the individual defect densities we first measured the total density by $\mathrm{cw}$ EPR at room temperature to calibrate relative signal intensities obtained by pulsed EPR. Using this procedure we can determine the absolute density of each individual defect type by integrating the disentangled EPR spectra. Figure 2(c) shows that annealing reduces defect densities by a factor of 2.5(9) (type I) and 11(9) (type II). We find that about 21(9)\% of all metastable defects are of type I while $79(9) \%$ are of type II. At this point it is important to highlight that we observe both types of defects in a variety of light-soaked samples deposited under different PECVD conditions (data not shown). The hereobserved effect is, therefore, a general feature of the SWE. A further interesting observation is that reversing lightinduced degradation by annealing shifts the whole EPR spectrum by $0.2(1) \mathrm{mT}$ to higher magnetic field values [see Fig. 1(a)]; i.e., the mean $g$ value shifts from 2.0053(2) to 2.0050(2), an effect which is currently not understood. 

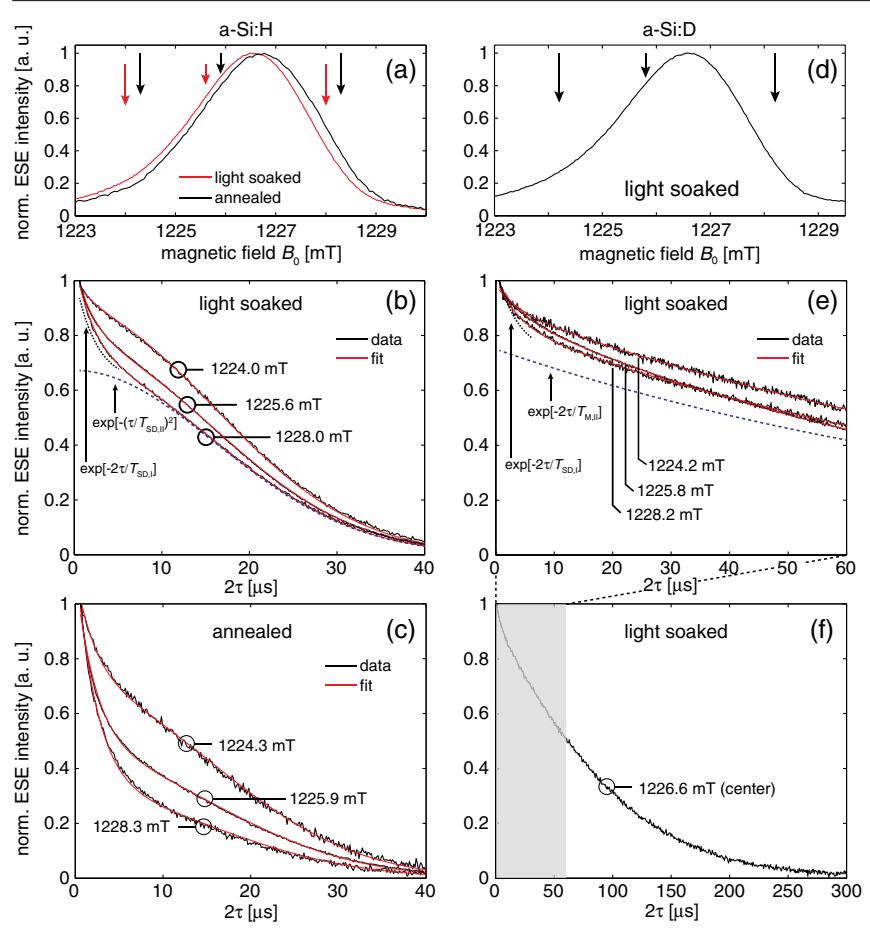

FIG. 1 (color online). (a) Normalized FSE-EPR spectra of defects in light-soaked (red) and annealed (black) $a-\mathrm{Si}: \mathrm{H}$ ( $\tau=300 \mathrm{~ns}$ ). (b),(c) Normalized ESE decays (black) of lightsoaked [annealed] $a-\mathrm{Si}: \mathrm{H}$ at magnetic-field positions marked by arrows in (a). Fits using a model described in the text are shown in red. Dashed lines show the ESE relaxation of the individual defect types. (d) Normalized FSE-EPR spectrum of light-soaked $a$-Si:D ( $\tau=300 \mathrm{~ns})$. (e) Normalized ESE decays of metastable defects in $a$-Si:D at different magnetic-field positions [marked by arrows in (d)]. Fits using a model described in the Supplemental Material [36] are shown in red. Dashed lines show the ESE relaxation of the individual defect types. (f) ESE decay of metastable defects in $a$-Si:D plotted for larger $\tau$ values [magnetic-field position in the center of the EPR spectrum shown in (d)]. All spectra were recorded at the same microwave frequency.

We have seen above that the ESE decay of defect type I is not affected by exchanging isotopes $\left({ }^{1} \mathrm{H}\right.$ with $\left.{ }^{2} \mathrm{D}\right)$, which implies that ${ }^{1} \mathrm{H}$-induced SD is not the dominant relaxation mechanism. Other relaxation mechanisms relying on spin flips induced by spin-lattice relaxation $\left(T_{1}\right)$ [30] can be excluded, since ESE decays are temperature independent below $60 \mathrm{~K}$ while $T_{1}$ exhibits a steep temperature dependence (see the Supplemental Material [36]). Temperatureindependent mechanisms such as ID or direct flip flops [39] can also be excluded, since the ESE decay of defects in $a-\mathrm{Si}: \mathrm{H}$ does not depend on microwave pulse flip angle as described in the Supplemental Material [36]. Instead we propose that indirect flip flops of adjacent electron-spin pairs lead to the observed ESE decay. If the dipolar coupling between pairs of $B$ spins (marked black) is sufficiently large, they carry out flip flops [see Fig. 2(d)] changing their spin orientation in a random manner [39]. This modulates the magnetic field felt by the $A$ spin (marked red) leading to SD. This ESE relaxation process is independent of temperature and flip angle and is in agreement with our experimental observations. It shall be noted that the indirect flip-flop rate is only temperature independent if the thermal energy is larger than the Zeeman energy, i.e., the spin ensemble is not polarized [40,41], a condition that is fulfilled here (the Zeeman energy at $1.2 \mathrm{~T}$ corresponds to $1.6 \mathrm{~K}) . T_{\mathrm{SD}, \mathrm{I}}$ is, therefore, only a function of flip-flop rate $(W)$ and dipolar coupling between the spin pair and the observed spin [39]. In addition, $W$ depends on the spatial arrangement of spins and spin-spin interactions within clusters consisting of two or more spins have to be considered for this relaxation mechanism [39]. Hence, analytic expressions for the ESE decay are currently not available. However, we can compare our case to a similar spin system consisting of ${ }^{31} \mathrm{P}$ donors in crystalline $\mathrm{Si}$, which was recently investigated by experiment and theory $[42,43]$. It was shown that indirect flip flops between electron spins of randomly distributed donors with a concentration of $N \approx 10^{16} \mathrm{~cm}^{-3}$ lead to a temperature independent ESE relaxation time constant of $500 \mu \mathrm{s}$ [42]. As this is two orders of magnitude larger than our result and the average spin concentration is roughly the same, we conclude that defects of type I are not randomly distributed. Instead, we propose that defects form clusters in which the locally small interspin distances lead to a large dipolar coupling and to a drastic increase in indirect flipflop rate. For $\mathrm{P}$ donors in $\mathrm{Si}$, Tyryshkin et al. [42] found a linear relationship between $N$ and relaxation rate: $1 / T_{2}\left[\mathrm{~s}^{-1}\right] \approx 10^{-14} N\left[\mathrm{~cm}^{-3}\right]$. This result is supported by recent theoretical studies [43] and can also be understood in a simple dipole model originally established by Bloch for NMR [44]. Applying Bloch's model to electron spin-spin relaxation gives $1 / T_{2}=\left(\mu_{0} \mu^{2} / 2 h r^{3}\right)$, where $\mu$ is the electron magnetic moment and $r$ is the interdipole distance. Replacing $r$ with a density $N$ of a random distribution, we arrive at $1 / T_{2}\left[\mathrm{~s}^{-1}\right] \approx 8 \times 10^{-14} N\left[\mathrm{~cm}^{-3}\right]$, which is in good agreement with the experimental data of $\mathrm{P}$-doped $\mathrm{Si}$ considering the simplicity of Bloch's theory. Extrapolating the data points reported in Ref. [42] to $T_{\mathrm{SD}, \mathrm{I}}=2.4(1) \mu \mathrm{s}$, allows us to asses a local defect concentration. We obtain $N_{\text {loc }} \approx 4 \times 10^{19} \mathrm{~cm}^{-3}$ which corresponds to an average distance of $D=1.6 \mathrm{~nm}$. This result indicates that $N_{\text {loc }}$ is about 3 orders of magnitude larger than the average defect concentration. For $a-\mathrm{Si}: \mathrm{H}$, the existence of highly defective surface layers and a nonuniform defect distribution in the bulk after light soaking due to a nonuniform carrier profile have been reported [34]. However, these findings unlikely explain our results. The bulk defect distribution in our samples is significantly more uniform compared to Ref. [34] because we filter out blue light while Ref. [34] uses white light. Also metastable defects of type I unlikely stem from a highly defective surface layer, since Zhou et al. [34] find that the defect density in the surface layer is much less sensitive to light soaking compared to the bulk defect density. We, therefore, attribute the observed drop in defect density by a factor of 2.5(9) after annealing to the bulk 

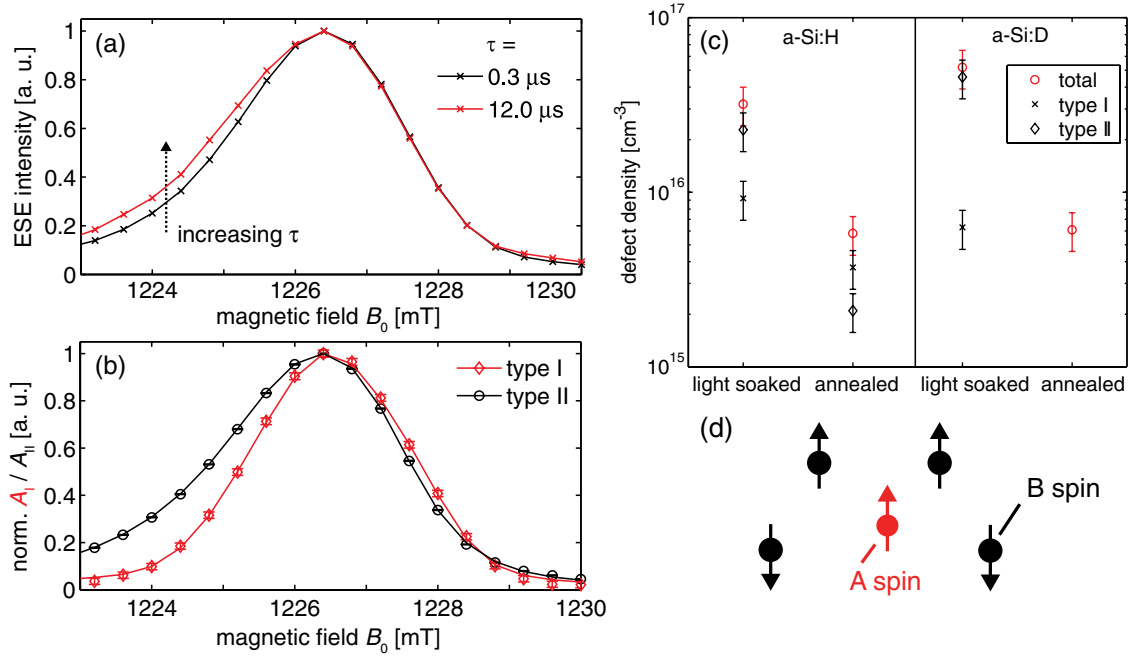

FIG. 2 (color online). (a) Normalized FSE-EPR spectra of light-soaked $a$-Si:H recorded at two different pulse delays $\tau$. (b) Normalized EPR spectra extracted from fitting ESE decays (see Fig. 1). (c) Total and partial defect densities in the light-soaked state and the annealed state. (d) Illustration of ESE relaxation by indirect flip-flops of neighboring B spin pairs.

defect density [see Fig. 2(c)]. In addition, our estimate for $N_{\text {loc }}$ is much higher compared to a typical defect density in a defective surface layer $\left(10^{18} \mathrm{~cm}^{-3}[45]\right)$. We, therefore, relate the high $N_{\text {loc }}$ to the inhomogeneous atomic network of $a-\mathrm{Si}: \mathrm{H}$. As reviewed in the introduction, $a-\mathrm{Si}: \mathrm{H}$ contains a large number of microvoids with a size of about 1-2 nm. When defects are concentrated on the inner surface of microvoids their distance is on average 1-2 nm, which reasonably well agrees with the average distance in defect clusters estimated from the ESE decay $(D=1.6 \mathrm{~nm})$.

Carlson [26] proposed that light-induced degradation is linked to $\mathrm{H}$ motion at internal surfaces of microvoids, which are reconstructed similar to the (100) surface of crystalline $\mathrm{Si}$ $[46,47]$. In the following we shall recapture the most important features of this model and Fig. 3 provides a corresponding illustration on the atomic level. The model states that weak Si-Si bonds of dimers trap free holes $\left(\mathrm{h}^{+}\right)$ leading to $\mathrm{H}$ motion, which eventually causes a rupture of dimeric Si-Si bond reconstruction and the formation of dangling bonds. Recent observations of fast light-induced degradation of $a-\mathrm{Si}: \mathrm{H} / c$-Si interfaces support this mechanism [48]. Considering the model by Carlson, metastable defects are created at internal surfaces of microvoids and it is reasonable to assume that several defects will be created leading to spin clustering if the microvoid is of sufficient size. We, therefore, propose that defects of type I are related to microvoids. As inferred from ID experiments, defects of type II are randomly distributed. However, we cannot decide whether defect type II is also associated with microvoids, as suggested recently (see Ref. [49]).

Above, we found that for $a-\mathrm{Si}: \mathrm{H}$ about 21(9)\% of all metastable defects are clustered (type I) and 79(9)\% are isolated (type II). In the annealed state both populations are roughly equal [see Fig. 2(c)]. This shows that clustered metastable defects, potentially associated with microvoids, play a significant role although they are only partially responsible for light-induced degradation. The reason why there are more isolated defects in the light-soaked state than clustered defects is not completely understood, but might be related to a higher abundance of precursor sites (weak $\mathrm{Si}-\mathrm{Si}$ bonds) available in the bulk of the $a$-Si:H film, while microvoids and their associated weak $\mathrm{Si}-\mathrm{Si}$ bonds are less abundant.

Figure 2(b) showed that defects of type I and II also differ in their EPR spectra, where type I exhibits a narrower spectrum compared to type II. This can be explained by exchange narrowing, where the exchange interaction in spin clusters averages the anisotropic $g$ tensor [50,51].

In summary, we investigated defects in light-soaked and annealed $a-\mathrm{Si}: \mathrm{H}(\mathrm{D})$ by time-domain pulsed EPR spectroscopy. We distinguished two defect types (I, II) by their

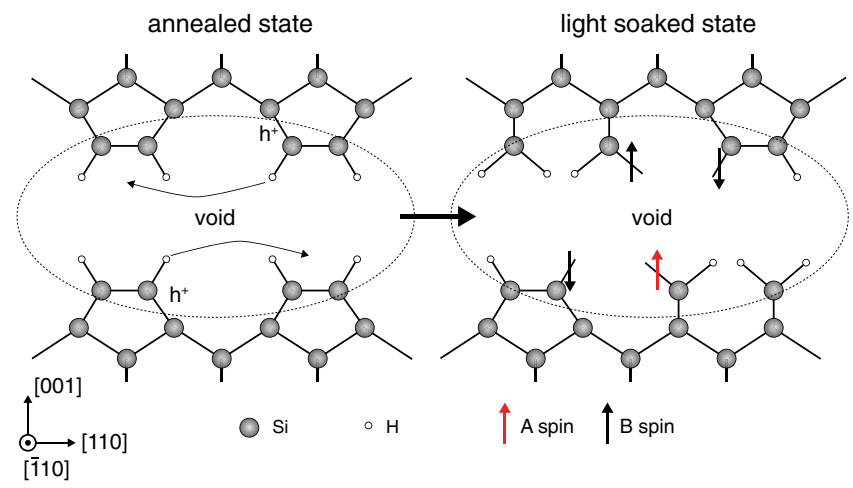

FIG. 3 (color online). Light-induced defect formation at internal surfaces of microvoids: schematic of the atomic structure in the light-soaked and annealed state after Carlson [26]. Atomic positions are projected onto the (110) plane and orientations corresponding to single-crystalline $\mathrm{Si}$ are shown in the lower left corner. Arrows indicate $\mathrm{H}$ motion and $\mathrm{h}^{+}$denotes a hole trapped at weak $\mathrm{Si}$-Si bonds. The formation of spin clusters in the lightsoaked state leads to indirect flip flops of $B$ spins (black) causing a rapid decoherence of observed $A$ spins (red). 
ESE relaxation properties. ESE relaxation of defect type I is dominated by indirect flip flops between dipolar-coupled spins, indicating that these defects occur in clusters. We propose that defect clusters are associated with microvoids. In contrast, defects of type II are randomly distributed and exhibit an ESE relaxation, which is determined by ${ }^{1} \mathrm{H}$ nuclear-spin flip flops. Both defect populations are present in the annealed as well as in the light-soaked state and both contribute to light-induced degradation. While isolated defects [79(9)\%] constitute the majority of metastable defects, the portion of clustered metastable defects [21 (9)\%], potentially associated with microvoids, is also significant. This result indicates that microvoids play a role in light-induced degradation of $a-\mathrm{Si}: \mathrm{H}$.

Financial support from BMBF (EPR-Solar network project 03SF0328) and the German Israeli Science Foundation (Grant No. 1032) is acknowledged. M. F. is thankful for support from the Alexander von Humboldt Foundation.

*Present address: Department of Chemistry and Biochemistry, University of California, Santa Barbara, California 93106, USA.

matthias.fehr@helmholtz-berlin.de

†alexander.schnegg@helmholtz-berlin.de

[1] D. L. Staebler and C. R. Wronski, Appl. Phys. Lett. 31, 292 (1977).

[2] D. L. Staebler and C. R. Wronski, J. Appl. Phys. 51, 3262 (1980).

[3] M. Stutzmann, W. B. Jackson, and C. C. Tsai, Phys. Rev. B 32, 23 (1985).

[4] H. Dersch, J. Stuke, and J. Beichler, Appl. Phys. Lett. 38, 456 (1981).

[5] M. Stutzmann and D. K. Biegelsen, Phys. Rev. B 40, 9834 (1989).

[6] M. Fehr, A. Schnegg, B. Rech, K. Lips, O. Astakhov, F. Finger, G. Pfanner, C. Freysoldt, J. Neugebauer, R. Bittl, and C. Teutloff, Phys. Rev. B 84, 245203 (2011).

[7] M. J. Powell, S. C. Deane, and R. B. Wehrspohn, Phys. Rev. B 66, 155212 (2002).

[8] W. B. Jackson, Phys. Rev. B 41, 10257 (1990).

[9] S. B. Zhang, W. B. Jackson, and D. J. Chadi, Phys. Rev. Lett. 65, 2575 (1990).

[10] C. Longeaud, D. Roy, and O. Saadane, Phys. Rev. B 65, 085206 (2002).

[11] M. Stutzmann, Mater. Res. Soc. Symp. Proc. 467, 37 (1997).

[12] H. Fritzsche, Annu. Rev. Mater. Res. 31, 47 (2001).

[13] H. M. Branz, Phys. Rev. B 59, 5498 (1999).

[14] M. Cardona, Phys. Status Solidi B 118, 463 (1983).

[15] A. H. Mahan, D. L. Williamson, B. P. Nelson, and R. S. Crandall, Phys. Rev. B 40, 12024 (1989).

[16] A. H. M. Smets and M. C. M. van de Sanden, Phys. Rev. B 76, 073202 (2007).

[17] J. Melskens, A. H. M. Smets, M. Schouten, S. W. H. Eijt, H. Schut, and M. Zeman, IEEE J. Photovoltaics 3, 65 (2013).

[18] W. Beyer, W. Hilgers, P. Prunici, and D. Lennartz, J. NonCryst. Solids 358, 2023 (2012).

[19] J. Baum, K. K. Gleason, A. Pines, A. N. Garroway, and J. A. Reimer, Phys. Rev. Lett. 56, 1377 (1986).
[20] Y. Wu, J. T. Stephen, D. X. Han, J. M. Rutland, R. S. Crandall, and A. H. Mahan, Phys. Rev. Lett. 77, 2049 (1996).

[21] Z. Remeš, M. Vaněček, A. H. Mahan, and R. S. Crandall, Phys. Rev. B 56, R12710 (1997).

[22] X. Zou, Y. C. Chan, D. P. Webb, Y. W. Lam, Y. F. Hu, C. D. Beling, S. Fung, and H. M. Weng, Phys. Rev. Lett. 84, 769 (2000).

[23] A. H. M. Smets, W. M. M. Kessels, and M. C. M. van de Sanden, Appl. Phys. Lett. 82, 1547 (2003).

[24] S. Guha, J. Yang, S. J. Jones, C. Yan, and D. L. Williamson, Appl. Phys. Lett. 61, 1444 (1992).

[25] E. Bhattacharya and A. H. Mahan, Appl. Phys. Lett. 52, 1587 (1988).

[26] D. E. Carlson, Appl. Phys. A 41, 305 (1986).

[27] J. R. Klauder and P. W. Anderson, Phys. Rev. 125, 912 (1962).

[28] W. B. Mims, Phys. Rev. 168, 370 (1968).

[29] P. Hu and S. R. Hartmann, Phys. Rev. B 9, 1 (1974).

[30] K. M. Salikhov, S. A. Dzuba, and A. M. Raitsimring, J. Magn. Reson. 42, 255 (1981).

[31] R. de Sousa and S. Das Sarma, Phys. Rev. B 67, 033301 (2003).

[32] A. Shah, Thin-Film Silicon Solar Cells (EPFL Press, CRC Press, Lausanne, 2010).

[33] L. Xiao, O. Astakhov, and F. Finger, Jpn. J. Appl. Phys. 50, 071301 (2011).

[34] J. H. Zhou, M. Kumeda, and T. Shimizu, Phys. Rev. B 53, 7267 (1996).

[35] S. Yamasaki and J. Isoya, J. Non-Cryst. Solids 164-166, Part 1, 169 (1993).

[36] See Supplemental Material at http://link.aps.org/ supplemental/10.1103/PhysRevLett.112.066403 for further information about sample preparation, EPR spectroscopy as well as temperature, flip-angle and magnetic-field dependence of ESE decays.

[37] J. Isoya, S. Yamasaki, A. Matsuda, and K. Tanaka, Philos. Mag. B 69, 263 (1994).

[38] S. Yamasaki and J. Isoya, Optoelectron. Devices Technol. 9, 345 (1994).

[39] V. V. Kurshev and T. Ichikawa, J. Magn. Reson. 96, 563 (1992).

[40] C. Kutter, H. P. Moll, J. van Tol, H. Zuckermann, J. C. Maan, and P. Wyder, Phys. Rev. Lett. 74, 2925 (1995).

[41] S. Takahashi, R. Hanson, J. van Tol, M. S. Sherwin, and D. D. Awschalom, Phys. Rev. Lett. 101, 047601 (2008).

[42] A. M. Tyryshkin, S. Tojo, J. J. L. Morton, H. Riemann, N. V. Abrosimov, P. Becker, H. J. Pohl, T. Schenkel, M. L. W. Thewalt, K. M. Itoh, and S. A. Lyon, Nat. Mater. 11, 143 (2011).

[43] W. M. Witzel, M. S. Carroll, A. Morello, L. Cywinski, and S. Das Sarma, Phys. Rev. Lett. 105, 187602 (2010).

[44] F. Bloch, Phys. Rev. 70, 460 (1946).

[45] J. Zhou, M. Kumeda, and T. Shimizu, Appl. Phys. Lett. 66, 742 (1995).

[46] T. Sakurai and H. D. Hagstrum, Phys. Rev. B 14, 1593 (1976).

[47] D. J. Chadi, Phys. Rev. Lett. 43, 43 (1979).

[48] S. De Wolf, B. Demaurex, A. Descoeudres, and C. Ballif, Phys. Rev. B 83, 233301 (2011).

[49] A. H. M. Smets, C. R. Wronski, M. Zeman, and M. C. M. van de Sanden, Mater. Res. Soc. Symp. Proc. 1245, 1245 (2010).

[50] P. W. Anderson and P. R. Weiss, Rev. Mod. Phys. 25, 269 (1953).

[51] R. Bachus, B. Movaghar, L. Schweitzer, and U. VogetGrote, Philos. Mag. B 39, 27 (1979). 